\title{
Cabergoline reduces 3-methoxytyramine in a SDHC patient with metastatic paraganglioma and prolactinoma
}

\author{
Ziad Hussein ${ }^{1} 1,2$, Marta Korbonits³, Stephanie E Baldeweg1,2 and Teng-Teng Chung1 \\ 1Department of Endocrinology, University College London Hospital, London, UK, 2Department of Medicine, University \\ College London, London, UK, and 'William Harvey Research Institute, Barts and the London School of Medicine, \\ Queen Mary University of London, London, UK
}

\section{Correspondence} should be addressed to T Chung

Email

teng-teng.chung@nhs.net

\section{Summary}

We observed a novel therapeutic response with cabergoline in a male patient with a dopamine-secreting head and neck paraganglioma (HNPGL), macroprolactinoma and germline succinate dehydrogenase $\mathrm{C}$ mutation (SDHC). The macroprolactinoma was treated with cabergoline which gave an excellent response. He was found to have raised plasma 3-methoxytyramine of $1014 \mathrm{pmol} / \mathrm{L}$ (NR: 0-180 pmol/L); but it was unclear if this was a drug-induced phenomenon from dopamine agonist (DA) therapy. Cabergoline was stopped for 4 weeks and the 3-methoxytyramine level increased significantly to $2185 \mathrm{pmol} / \mathrm{L}$, suggesting a biochemical response of his HNPGL. Subsequently, Gallium-68 Dotatate PET and MRI (Gallium-68 Dotatate PET/MRI) demonstrated a second lesion in the sacrum. Both the HNPGL and metastatic sacral deposit received external beam radiotherapy with a good biochemical and radiological response.

Conclusion: Our case report highlights the rare potential of germline SDHC mutations causing metastatic paraganglioma and concurrent pituitary tumours. Cabergoline treatment may lower elevated 3-methoxytyramine levels and, therefore, mask the biochemical evidence of metastatic disease but also may have therapeutic relevance in dopamine-secreting pheochromocytomas/paragangliomas (PPGLs).

\section{Learning points:}

- Several neuroendocrine tumours (NETs) express dopamine D2 and D4 receptors. In this case report, cabergoline significantly reduced plasma 3-methoxytyramine level in a patient with functional HNPGL. Cabergoline might have therapeutic relevance in dopamine-secreting PPGLs.

- Paragangliomas associated with SDHC mutation classically present with asymptomatic non-functional HNPGL and have rare metastatic potential.

- The association of pheochromocytoma or paraganglioma and pituitary adenoma is now a well-described rare association ( $<1 \%)$, designated as the three $\mathrm{P}$ association. While the three $\mathrm{P}$ association is most commonly seen with succinate dehydrogenase $B$ and $D$ mutations, it has also been described in patients with SDHA and SDHC mutations.

- Cabergoline treatment may lower elevated 3-methoxytyramine levels and mask the biochemical evidence of metastatic disease. Regular functional imaging with Gallium-68 Dotatate PET/MRI provides better evidence of metastatic disease. 


\section{Background}

HNPGL are NETs, arising from parasympathetic ganglia in the neck and skull base. These tumours present mainly with non-specific symptoms, including painless neck masses compressing the cranial nerves or invading the skull base. It has been reported that up to one-third of HNPGL patients have biochemically active tumours producing 3-methoxytyramine (1), which is a metabolite of dopamine. The increased production of dopamine has been shown to be an independent marker for metastatic disease (2).

Several NETs exhibit dopamine D2 and D4 receptors, including pheochromocytoma (PCC) and paraganglioma (PGL) $(3,4)$, suggesting that these tumours might be amenable to dopaminergic therapy.

In our case report, we present a possible therapeutic response in a patient with dopamine-secreting HNPGL and a pituitary adenoma associated with germline SDHC mutation. We observed a novel association of decreased plasma 3-methoxytyramine with cabergoline administered for pituitary macroprolactinoma. This case is also unique, it highlights the rare association of metastatic disease from HNPGL with pituitary adenoma and SDHC mutation.

\section{Case presentation}

A 64-year-old man presented in 1993 with swallowing difficulties caused by HNPGL. He underwent two transtemporal resections in 1993 and 2000; complete excision of the tumour was not possible due to its size and proximity to crucial neck structures. The residual disease remained stable and was monitored with regular MRI under the Ear, Nose and Throat surgeons. In 2008, a surveillance MRI demonstrated an incidental pituitary macroadenoma (Fig. 1). Apart from occasional headaches, he had no history of hypertension, palpitations or sweating to suggest catecholamine overproduction. On examination in 2008, his blood pressure was $112 / 78 \mathrm{mmHg}$ and pulse rate of 90 b.p.m. He had normal visual fields, but residual right-sided 7th, 9th, 10th, 11thand 12th cranial nerves deficits due to previous surgeries.

\section{Investigations}

His pituitary profile showed a markedly raised prolactin of $43000 \mathrm{mIU} / \mathrm{L}$ (NR: $86-324 \mathrm{mIU} / \mathrm{L}$ ). The patient was then referred to endocrinology and started on cabergoline $0.5 \mathrm{mg}$ weekly; the macroprolactinoma demonstrated an excellent response with normalisation of serum

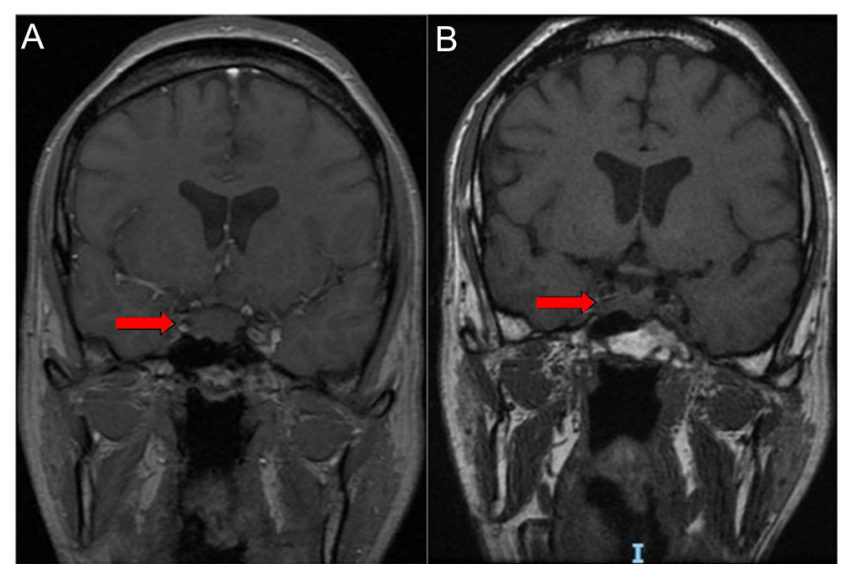

Figure 1

(A) T1 weighted MRI of the head (coronal view) performed in 2008 showed an incidental pituitary macroadenoma (red arrow). (B) T1 weighted MRI of the head (coronal view) in 2011 showed the macroadenoma responded well to dopamine agonist therapy with a reduction in size.

prolactin within 1 year. MRI scan in 2011 (Fig. 1) showed a significant reduction in prolactinoma size from baseline; the cabergoline dose was subsequently reduced to $0.25 \mathrm{mg}$ weekly.

Interestingly, his family history revealed a brother with a diagnosis of HNPGL and a maternal cousin with non-functioning pituitary adenoma (NFPA), both were in their fifties. Genetic testing found that our index case harboured a novel missense variant in $S D H C$ gene c.380A $>\mathrm{G}$ (p.His127Arg) with a gnomAD minor allele frequency of 0.0000315 . The same genetic alteration was found in his brother with paraganglioma. The proband's mother, aunt, sister, two nephews and one niece are carriers of the same mutation but have no clinical manifestation to date while the cousin with NFPA had no SDHC gene alteration detected, he was considered to represent a phenocopy. No mutations were identified in multiple endocrine neoplasia 1 gene (MEN 1) and Aryl hydrocarbon receptor interacting protein (AIP) gene in the proband or his family members. Family pedigree is shown in Fig. 2.

In May 2012, he complained of occasional headaches, but no other symptoms. His PGL showed radiological evidence of progression and compression of the right internal carotid artery. At this stage, the biochemical assessment of the PGL was performed for the first time. He had normal plasma metanephrine at $377 \mathrm{pmol} / \mathrm{L}$ (NR: $80-510 \mathrm{pmol} / \mathrm{L}$ ) and normetanephrine at $284 \mathrm{pmol} / \mathrm{L}$ (NR: 120-1180 pmol/L) but raised 3-methoxytyramine concentration at $1014 \mathrm{pmol} / \mathrm{L}$ (NR: 0-180 pmol/L). We questioned if the isolated rise in 3-methoxytyramine was a drug-induced phenomenon caused by DA. Cabergoline was temporarily stopped for 4 weeks; plasma 


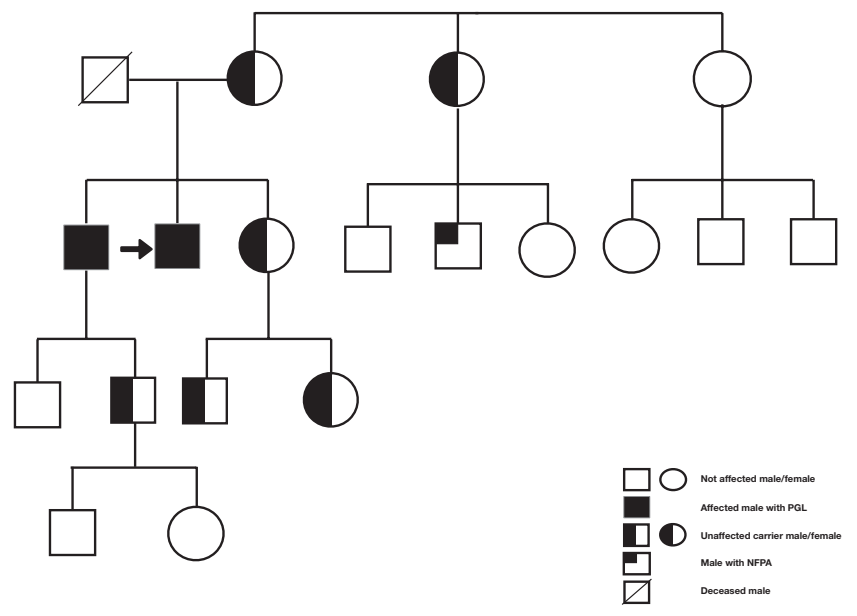

Figure 2

This family pedigree chart demonstrates the family history of the index patient who is marked with an arrow. NFPA, non-functioning pituitary adenoma; $\mathrm{PGL}$, paraganglioma.

3-methoxytyramine level increased substantially to 1965 $\mathrm{pmol} / \mathrm{L}$ and $2185 \mathrm{pmol} / \mathrm{L}$ at 2 and 4 weeks of ceasing the drug, respectively. After restarting cabergoline, plasma 3-methoxytyramine dropped down and returned to the previously raised level (Fig. 3). Elevated 3-methoxytyramine was concerning as the metabolite is considered a marker for metastatic disease in PPGLs (2). Subsequently, functional imaging with Gallium-68 Dotatate PET/MRI demonstrated avid uptake at his residual neck disease and a small focus of uptake in the sacral body (S2) suggestive of metastatic focus (Fig. 4). To further characterise the lesion, an MRI of the sacrum with diffusion sequences showed a $7 \times 12 \mathrm{~mm}$ lesion compatible with PGL deposit. A sacral biopsy was not attempted because of the small size and high risk of neural injury.

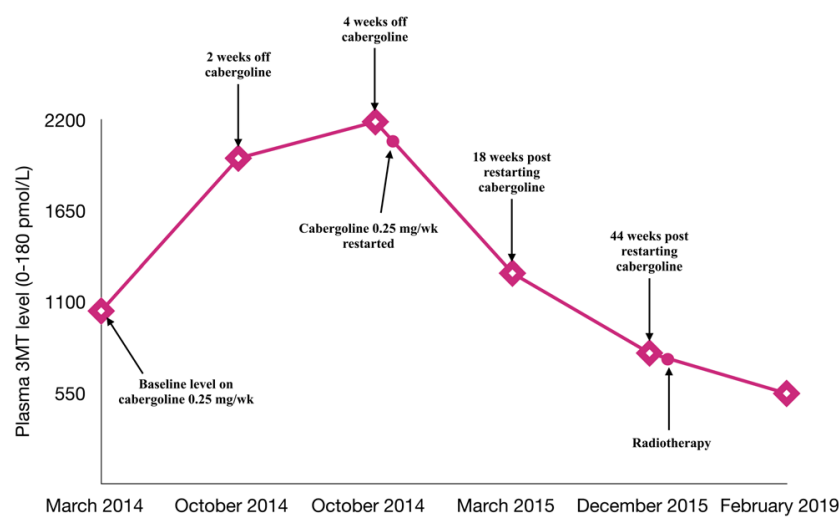

Figure 3

Plasma 3-methoxytyramine concentrations with dopamine agonists and radiotherapy. 3-methoxytyramine concentrations doubled after stopping cabergoline and returned to raised earlier concentration after re-starting therapy. 3MT, 3-methoxytyramine; mg, milligram; wk, week.

\section{Treatment}

The patient continued on cabergoline $0.25 \mathrm{mg}$ weekly and received 50.4 Gray of fractionated external beam radiotherapy to both skull base and the sacral lesions in December 2015.

\section{Outcome and follow-up}

Five years post-irradiation, the patient remained stable. Plasma 3-methoxytyramine reduced to $489 \mathrm{pmol} / \mathrm{L}$. Gallium-68 Dotatate PET/MRI scan in 2020 demonstrated stable disease at the skull base and sacrum. Prolactin remained within reference range on cabergoline $0.25 \mathrm{mg}$ once weekly. MRI pituitary continued to show regressing pituitary adenoma with the plan to repeat surveillance scanning in 5 years. Annual measurement of plasma metanephrines and periodic radiological assessment (MRI/ Gallium-68 Dotatate PET/MRI) are in place to monitor PGL activity and progression of metastatic disease. His asymptomatic carrier family members are under regular screening follow-up with their local endocrinologists.

\section{Discussion}

Cabergoline is an ergot-derived dopamine 2 (D2) receptor agonist. Some studies have reported the expression of D2 receptors in various NETs including gastrointestinal, pancreatic and bronchial NETs and PCC $(3,5)$. Saveanu et al. (4) studied 52 patients with PPGLs including patients with $S D H B$ and $S D H D$ mutation, all tumours expressed high levels of D2 mRNA as surrogate evidence of D2 receptors. Their data showed that D2 is expressed in PGL at high levels (though not as high as PCC). It has been shown that the activation of dopamine receptors inhibits endogenous dopamine release with the subsequent reduction of dopamine metabolism to its metabolites including 3-methoxytyramine (6). The evidence of antisecretory and possibly antiproliferative effect of DA has been seen in ACTH-secreting bronchial NETs (5) and metastatic islet cell tumours (7). However, the effect of D2 receptor agonists remains uncertain in PPGLs but may have clinical relevance in treating dopamine secreting PPGLs.

Exclusive dopamine-secreting PPGLs differ in the clinical presentation when compared with other catecholamine-producing counterparts. These tumours are typically associated with extra adrenal location and have more metastatic potential than other catecholamine-secreting tumours (2). This behaviour has been attributed to poor cellular differentiation and 

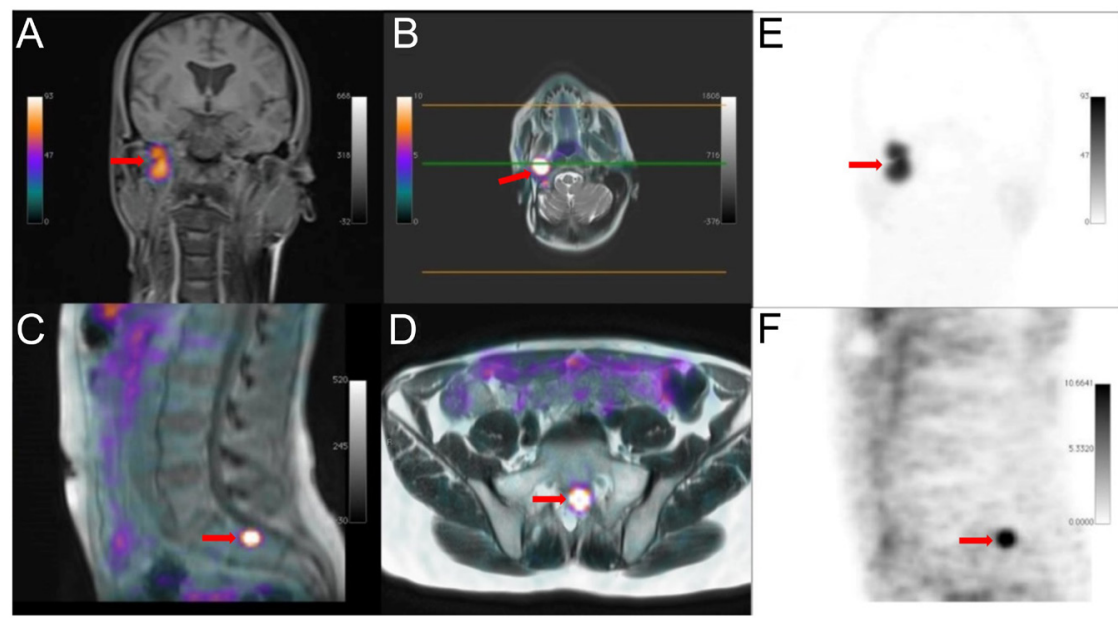

Figure 4

(A and B) PET and MRI of the head and neck (A: coronal view, B: axial view) demonstrated avid uptake at the right-sided residual paraganglioma lesion (red arrows). (C and D) PET/MRI scan of lumbosacral region (C: sagittal view, D: axial view) demonstrated avid uptake at the site of metastatic paraganglioma in the sacral body (S2) (red arrows). (E) Imaging showed increased Gallium-68 Dotatate uptake at the residual head and neck paraganglioma (red arrow) and (F) showed increased Gallium-68 Dotatate in the sacral region (red arrow).

delayed clinical presentation due to the absence of symptoms. Elevated plasma 3-methoxytyramine can be considered as an independent marker of metastatic potential and can increase up to five-fold in patients with metastasis compared to those without (2). Our patient was normotensive and asymptomatic from dopamine excess; the effect of cabergoline on his 3-methoxytyramine is clearly demonstrated. It is unclear if the use of DAs had a definite effect on his tumour control/progression or likely response to radiotherapy. To date, no further metastasis has been detected. It would be of interest to explore the antiproliferative effects of DAs in dopamine-secreting PPGLs.

The association of PCC or PGL and pituitary adenoma is now a well-described rare $(<1 \%)$ association, designated as the three $\mathrm{P}$ association or '3PA'. PGLs associated with SDHC mutation classically present with isolated asymptomatic, non-functional HNPGL. The first case of metastatic PGL with distant metastases caused by SDHC mutation was described in 2014 by Bickman et al. (8). Subsequently, others have also published metastatic PGL in SDHC $(9,10)$. In our patient, the relationship of pituitary tumour and SDHC remains undefined, as loss of heterozygosity or a second somatic mutation study is not available as the macroprolactinoma was treated medically.

Our case highlights the rare potential of germline SDHC mutations causing metastatic HNPGL with concurrent prolactin-secreting pituitary tumours and an interesting effect on 3-methoxytyramine with the use of DA. Cabergoline treatment may lower elevated 3-methoxytyramine levels but equally, it could mask the biochemical evidence of metastasis. Therefore, Gallium-68 Dotatate PET/MRI scanning provides better evidence of metastatic disease.

\section{Declaration of interest}

The authors declare that there is no conflict of interest that could be perceived as prejudicing the impartialsity of the research reported.

\section{Funding}

This research did not receive any specific grant from any funding agency in the public, commercial or not-for-profit sector.

\section{Patient consent}

Patient consent has been obtained.

\section{Patient's perspective}

The patient reviewed the manuscript and dose not want to change or amend the report

\section{Author contribution statement}

Ziad Hussein did a literature search and wrote up the manuscript. Marta Korbonits and Stephanie E Baldeweg reviewed the manuscript. Teng-Teng Chung is the consultant in charge for the patient, significantly contributed to the manuscript.

\section{References}

1 Van Der Horst-Schrivers ANA, Osinga TE, Kema IP, Van Der Laan BFAM \& Dullaart RPF. Dopamine excess in patients with head and neck paragangliomas. Anticancer Research 201030 5153-5158. (available at: http://www.ncbi.nlm.nih.gov/pubmed/21187504).

2 Eisenhofer G, Lenders JWM, Siegert G, Bornstein SR, Friberg P, Milosevic D, Mannelli M, Linehan WM, Adams K, Timmers HJ et al. Plasma methoxytyramine: A novel biomarker of metastatic pheochromocytoma and paraganglioma in relation to established risk factors of tumour size, location and SDHB mutation status. European Journal of Cancer 2012 [cited 2019 Apr 26] 48 1739-1749. (https://doi. org/10.1016/j.ejca.2011.07.016) (available at: http://www.ncbi.nlm.nih. gov/pubmed/22036874) 
3 Grossrubatscher E, Veronese S, Ciaramella PD, Pugliese R, Boniardi M, De Carlis L, Torre M, Ravini M, Gambacorta M \& Loli P. High expression of dopamine receptor subtype 2 in a large series of neuroendocrine tumors. Cancer Biology and Therapy 20087 1970-1978 (https://doi.org/10.4161/cbt.7.12.6957)

4 Saveanu A, Muresan M, De Micco C, Taieb D, Germanetti AL, Sebag F, Henry JF, Brunaud L, Enjalbert A, Weryha G et al. Expression of somatostatin receptors, dopamine $\mathrm{D} 2$ receptors, noradrenaline transporters, and vesicular monoamine transporters in 52 pheochromocytomas and paragangliomas. Endocrine-Related Cancer 201118 287-300 (https://doi.org/10.1530/ERC-10-0175)

5 Pivonello R, Ferone D, de Herder WW, Faggiano A, Bodei L, de Krijger RR, Lombardi G, Colao A, Lamberts SW \& Hofland LJ. Dopamine receptor expression and function in corticotroph ectopic tumors. Journal of Clinical Endocrinology and Metabolism 2007 [cited 2020 May 29] 92 65-69. (https://doi.org/10.1210/jc.2006-0728)

6 Ponzio F, Achilli G, Perego C \& Algeri S. Differential effects of certain dopaminergic drugs on the striatal concentration of dopamine metabolites, with special reference to 3-methoxytyramine. Neuroscience Letters 198127 61-67. (https://doi.org/10.1016/0304-3940(81)90206-8)
7 Pathak RD, Tran TH \& Burshell AL. A case of dopamine agonists inhibiting pancreatic polypeptide secretion from an islet cell tumor. Journal of Clinical Endocrinology and Metabolism 200489 581-584. (https://doi.org/10.1210/jc.2003-031039)

8 Bickmann JK, Sollfrank S, Schad A, Musholt TJ, Springer E, Miederer M, Bartsch O, Papaspyrou K, Koutsimpelas D, Mann WJ et al. Phenotypic variability and risk of malignancy in $S D H C$-Linked paragangliomas: Lessons From three unrelated cases with an identical germline mutation. Journal of Clinical Endocrinology and Metabolism 201499 E489-E496. (https://doi.org/10.1210/jc.2013-3486)

9 Isaacson B, Bullova P, Frone M, Click A, Hamplova B, Rabaglia J, Woodruff S, Nwariaku F, Kathuria A, Pacak K et al. An aggressive temporal bone sdhc paraganglioma associated with increased HIF- $2 \alpha$ signaling. Endocrine Practice 201622 190-195 (https://doi.org/10.4158/ EP15889.OR

10 Ong RKS, Flores SK, Reddick RL, Dahia PLM \& Shawa H. A unique case of metastatic, functional, hereditary paraganglioma associated with an SDHC germline mutation. Journal of Clinical Endocrinology and Metabolism 2018103 2802-2806. (https://doi.org/10.1210/jc.201701302)

Received in final form 22 April 2021

Accepted 10 May 2021 